\title{
Genetic Polymorphisms of Peroxisome Proliferator-Activated Receptors and the Risk of Cardiovascular Morbidity and Mortality in a Community-Based Cohort in Washington County, Maryland
}

\author{
L. Gallicchio, ${ }^{1}$ Bindu Kalesan, ${ }^{1}$ Han-Yao Huang, ${ }^{2}$ Paul Strickland, ${ }^{3}$ \\ Sandra C. Hoffman, ${ }^{2}$ and Kathy J. Helzlsouer ${ }^{1}$ \\ ${ }^{1}$ Prevention and Research Center, Weinberg Center for Women's Health and Medicine, Mercy Medical Center, \\ 227 Street Paul Place, 6th Floor, Baltimore, MD 21202, USA \\ ${ }^{2}$ Department of Epidemiology, Johns Hopkins Bloomberg School of Public Health, 615 North Wolfe Street, \\ Baltimore, MD 21205, USA \\ ${ }^{3}$ Department of Environmental Health Sciences, Johns Hopkins Bloomberg School of Public Health, 615 North Wolfe Street, \\ Room E7535, Baltimore, MD 21205, USA
}

Correspondence should be addressed to L. Gallicchio, lgallic@mdmercy.com

Received 27 June 2007; Revised 15 September 2007; Accepted 2 October 2007

Recommended by Brian N. Finck

The primary aim of this study was to examine prospectively the associations between 5 peroxisome proliferator-activated receptor (PPAR) single nucleotide polymorphisms (SNPs) and cardiovascular morbidity and mortality in a community-based cohort study in Washington County, Maryland. Data were analyzed from 9364 Caucasian men and women participating in CLUE-II. Genotyping on 5 PPAR polymorphisms was conducted using peripheral DNA samples collected in 1989. The followup period was from 1989 to 2003. The results showed that there were no statistically significant associations between the PPAR SNPs and cardiovascular deaths or events. In contrast, statistically significant age-adjusted associations were observed for PPARG rs4684847 with both baseline body mass and blood pressure, and for PPARG rs709158, PPARG rs1175543, and PPARD rs2016520 with baseline cholesterol levels. Future studies should be conducted to confirm these findings and to explore the associations in populations with greater racial and ethnic diversity.

Copyright ( 2008 L. Gallicchio et al. This is an open access article distributed under the Creative Commons Attribution License, which permits unrestricted use, distribution, and reproduction in any medium, provided the original work is properly cited.

\section{INTRODUCTION}

The peroxisome proliferator-activated receptors (PPARs) are part of a superfamily of ligand-activated transcription factors involved in fatty acid oxidation and lipid metabolism [1]. Three distinct isoforms of PPARs that are encoded by separate genes have been identified: PPAR- $\alpha, P P A R-\gamma$, and $P P A R-\delta$ [2]. The three isoforms play distinct physiological roles depending on their tissue distribution. PPAR- $\alpha$, which is expressed in the liver, heart, skeletal muscle, and kidney, regulates lipid and lipoprotein metabolism. PPAR$\gamma$ is expressed in white and brown adipose tissue and is involved in adipocyte differentiation, lipid storage, and glucose metabolism. PPAR- $\delta$ is expressed in many tissues and stim- ulates fatty acid oxidation $[2,3]$. Beyond these major roles, PPARs also have been shown to play a role in other biological processes, including the regulation of inflammatory and oxidative pathways [2].

PPARs are found in endothelial and vascular smooth muscle cells and have been shown to influence inflammatory, fibrotic, and hypertrophic responses in the heart and vascular wall [4]. Because of their location and their involvement in fatty acid oxidation, lipid metabolism, and inflammation, the role of PPARs in cardiovascular disease and risk factors of cardiovascular disease has been of great interest. In general, activation of the PPARs, both naturally and synthetically, is considered beneficial for cardiovascular health [2]. Both PPAR- $\alpha$ and PPAR- $\gamma$ play a role in modulating 
atherosclerosis; for example, PPAR- $\gamma$ activation may promote monocyte apoptosis, contributing to the stabilization of atherosclerotic lesions $[5,6]$. Further, clinical trials have shown that the use of pharmacological PPAR agonists such as fibrates (PPAR- $\alpha$ agonist) is antiatherogenic. Fibrates elevate high-density lipoprotein (HDL) levels, decrease low-density lipoprotein (LDL) and triglyceride levels, and reduce an individual's risk of experiencing a cardiac event [7].

A number of PPAR polymorphisms have been identified within the 3 PPAR isoforms and there is a considerable amount of literature on the associations between these polymorphisms and cardiovascular risk factors (reviewed in Cresci [7]). There are less data, however, on the associations between the PPAR polymorphisms and cardiovascular disease events (e.g., myocardial infarction (MI), cardiovascularrelated death). Further, findings with regard to these associations have been inconsistent. For example, a case-control study nested within the Physician's Health Study suggested that the PPARG Pro12Ala polymorphism, located in exon B of PPAR- $\gamma$, is associated with a reduced risk of MI [8]. In contrast, a recent publication using data from the Health Professionals Followup Study showed that male carriers of the Ala12 allele had an increased risk of MI or cardiac death [9] while other studies have observed no statistically significant association between PPARG Pro12Ala and cardiovascular events or death $[9,10]$. Thus, additional studies of these polymorphisms are necessary to help us better understand the role of PPAR genetics in cardiovascular disease especially in light of available pharmacological PPAR targeted agents.

The primary aim of this study was to examine prospectively the associations between 5 PPAR polymorphisms (4 in PPAR- $\gamma$ : rs4684847, rs709158, rs1175543, and rs1801282; and 1 in PPAR- $\delta$ : rs2016520) and cardiovascular morbidity and mortality in a community-based cohort study in Washington County, Maryland. As a secondary aim, we also examined the associations between the PPAR polymorphisms and cardiovascular risk factors.

\section{METHODS}

\subsection{Study sample}

In 1974 and 1989, two cohorts named CLUE I and CLUE II ("Give us a Clue to Cancer and Heart Disease") were established in Washington County, Maryland. CLUE I and CLUE II enrolled 20305 and 25081 Washington County residents, respectively. At baseline for both cohorts, participants provided informed consent, completed a brief questionnaire, and donated a blood sample. The questionnaire ascertained data on age, gender, marital status, education, height and weight (CLUE II only), cigarette smoking, and medication and vitamin supplement use within the 48 hours prior to blood donation. In addition, in both 1974 and 1989, blood pressure was measured by a study nurse with a blood pressure cuff while the participant was in a seated position. Blood pressure was assessed three times in succession and the third blood pressure value was recorded. In 1989, total cholesterol (nonfasting) was assayed. Individuals who donated blood to both CLUE I and CLUE II constitute the Odyssey cohort $(N$ $=8394)[11,12]$.

In addition to the Odyssey cohort, a CLUE II subcohort was selected for case-cohort studies that would be conducted using the CLUE II cohort data. The subcohort was identified by taking an approximate $10 \%$ age- and sex-stratified random sample of CLUE II participants who donated a blood specimen and were adult residents of Washington County, Maryland. Of the 2460 participants identified for the subcohort, 807 were also in the Odyssey cohort. Therefore, 10047 unique participants were part of either the Odyssey cohort or the randomly selected CLUE II subcohort.

Of the participants in the Odyssey Cohort and the CLUE II subcohort, DNA was successfully extracted from the buffy coat samples of 9960 individuals (99.1\%). DNA from these participants was genotyped for polymorphisms in genes controlling biological processes such as inflammation that have been associated with multiple diseases. For the study presented here, $5 \%$ of the Odyssey and subcohort participants who had no data on all of the chosen PPAR SNPs $(n=475)$ were excluded from the analysis. Further, all non-Caucasians ( $n=121$ ) were excluded from the analysis because previous studies have shown that race is an important effect modifier in investigations of polymorphisms and disease and there were not a sufficient number of non-Caucasians in the cohort to analyze the associations among this group. With the exception of race, excluded and included participants did not differ with respect to baseline characteristics. This study was approved by the Institutional Review Board of the Johns Hopkins Bloomberg School of Public Health.

\subsection{Outcome assessment}

\section{Mortality}

All participants were followed from the date of blood draw to the date of death or the end of follow up (August 31, 2003), whichever came first. In the CLUE cohorts, deaths are identified through daily searches of obituaries, cross-linkage with death certificates for Washington County, and through searches of the Social Security Administration for individuals aged 65 or older and the National Death Index. Cause of death is ascertained from the underlying cause on Maryland State death certificates as coded by state nosologists. Of specific interest in this study were cardiovascular disease deaths, for which the underlying cause was coded as ICD-9 390-459 or ICD-10 I00-I99. During the followup period, 2159 deaths were documented in the Odyssey cohort and the CLUE II subcohort, and of these, 791 (36.6\%) were cardiovascular deaths. Approximately 4\% $(n=334)$ of the Odyssey cohort and the CLUE II subcohort participants were lost to follow up. Since these individuals were not documented to have died during the followup period, they were considered alive at the end of follow up and censored at August 31, 2003.

\section{Morbidity}

Information on cardiovascular events was obtained using participant self-report beginning with questionnaires 
administered in 1996 and about every 2 years thereafter. On these questionnaires, participants were asked whether a "doctor had told them they ever had" a specific condition and at what age the condition was first diagnosed. The cardiovascular-related outcomes queried were: diabetes, high blood pressure, high cholesterol, heart attack (MI), angina pectoris, stroke, transient ischemic attack, peripheral artery disease, arrhythmia, and blood clots. Data were examined across the questionnaires for consistency; $5 \%$ of the participants had inconsistent data with regards to self-reported events. However, exclusion of these participants did not change the results and, therefore, these participants were not excluded. For this analysis, we examined any self-reported nonfatal cardiovascular event as an outcome. A nonfatal cardiovascular event was defined as consistent reporting from 1996 to 2003 of any one of the following cardiovascular conditions: $\mathrm{MI}$, angina, stroke, transient ischemic attack, peripheral arterial disease, arrhythmia, or blood clots. We also considered a composite variable including only MI, stroke, transient ischemic attack, and peripheral arterial disease; however, the results were similar to the composite variable including all seven outcomes and, therefore, the seven outcome variable was used in all analyses. Individual diagnoses were also examined separately. Because of the inconsistency in the collection of data on the age at which a condition occurred as well as the large amount of missing data for the age variables, age at diagnosis data were not used in the analysis.

\subsection{Genotyping}

The PPAR SNPs analyzed in this study were a part of a larger group of 210 SNPs selected for investigation within the Odyssey cohort. SNPs were selected based on the following criteria: (a) the minor allele frequency was estimated to be $\geq 5 \%$ among Caucasians in the published literature or databases; (b) the polymorphism was in a gene of known or of promising importance in the development of cancer, cardiovascular diseases, and/or longevity; and (c) the polymorphism was either known to be functional or was likely to alter function based on the published literature. PPAR polymorphisms selected for analysis were rs2016520 (PPARD Ex4 + 15C > T), rs709158 (PPARG IVS9 + 4523A > G), rs1175543 (PPARG IVS9 + 7780A > G), rs1801282 (PPARG Pro12Ala), and rs4684847 (PPARG IVS3-6622C > T). To note, none of the other 210 SNPs selected for investigation in the Odyssey cohort were located in PPAR- $\alpha$, PPAR- $\gamma$, or PPAR- $\delta$.

DNA extracted from the preserved buffy coat samples collected in 1989 were used for genotyping. Within 6 hours of collection, the heparinized blood sample was centrifuged at $1500 \mathrm{~g}$ for 30 minutes at room temperature. Blood samples were separated into plasma, buffy coat, and red blood cells and frozen at $-70^{\circ} \mathrm{C}$ within 24 hours of collection. The buffy coat remained frozen until DNA extraction was performed. The DNA extraction procedures used the alkaline lysis method [13]. Genotyping was performed by Celera Genomics Co. (Rockville, Md, USA) for rs4684847, rs709158, and rs1175543 and by Applied Biosystems Inc. (Foster City, Calif, USA) for rs2016520 and rs1801282. All polymorphisms were genotyped using TaqMan technology. Labora- tory technicians were masked to disease status. Of the 9,364 participants in the analytic cohort, approximately $90 \%$ had data on all five genotypes; $6.7 \%$ had data on four, $2.4 \%$ had data on three, $0.7 \%$ had data on two, and $0.07 \%$ had data on only one.

\subsection{Statistical analysis}

The Hardy-Weinberg equilibrium for each SNP was tested by a goodness-of-fit approach. As reported in separate publication, all of the PPAR SNPs were in Hardy-Weinberg equilibrium [12]. The cohort characteristics were stratified by gender and compared using chi-square tests or student t-tests. Blood pressure at baseline was categorized into 3 groups independent of antihypertensive medication use as follows: normal, individuals with a systolic pressure less than 120 and diastolic pressure less than 80 ; hypertensive, those with systolic pressure greater than 140 or diastolic pressure greater than 90; and prehypertensive, those with a systolic pressure between 120 and 140 or diastolic pressure between 80 and 90 . The age-adjusted associations between the PPAR SNPs and cardiovascular risk factors (i.e., baseline BMI, cholesterol levels, blood pressure) were examined using logistic regression models. Age was adjusted for in all analyses as there were statistically significant age differences for several of the SNPs. Gender was not adjusted for in these analyses because it was not associated with SNP prevalence. Cox-proportional hazard ratios were calculated for both all-cause and cardiovascular mortality after adjustment for age. Since the nonfatal cardiovascular outcomes (including MI), followed a Poisson distribution, and age at diagnosis data were not used in the analysis, age-adjusted relative risks for nonfatal cardiovascular events and for only MI were obtained using Poisson regression methods; this type of analysis was also used when analyzing fatal and nonfatal outcomes combined. Premature death (both overall and due to cardiovascular disease) was also examined as an outcome variable and defined as death prior to the age of 65 . All analyses were done separately for the 5 SNPs and stratified by gender, diabetes diagnosis, and body mass index (BMI) at baseline. No differences in the risk estimates were observed in these strata and, therefore, only results for the entire cohort are presented.

To address the issue of multiple testing in this study, $P$ values for the associations between SNPs and the cardiovascular risk factors were adjusted for the false discovery rate utilizing Fisher's combination method using bootstrap resampling. All statistical analysis was carried out using SAS software, version 9.1 (SAS Institute, Inc., Cary, NC, USA). A two-sided $P$ value $\leq .05$ was considered statistically significant.

\section{RESULTS}

Baseline characteristics of the study sample, overall and by gender, are shown on Table 1. In 1989, males were significantly more likely than females to have some college education, to report being a current or former smoker, and to be categorized as prehypertensive or hypertensive. In addition, males had a significantly higher mean BMI than females. In 
TABle 1: Characteristics of study sample by gender, $N=9364, P$ value derived from $\chi 2$ test for categorical variables, Student's t-test for continuous variables.

\begin{tabular}{|c|c|c|c|c|}
\hline & Female $N=5776(\%)$ & Male $N=3588(\%)$ & Total (\%) & $P$ value \\
\hline Age, mean (SD) & $53.2(15.4)$ & $52.9(15.5)$ & $53.1(15.4)$ & .3974 \\
\hline Education & & & & .0029 \\
\hline$<12$ & 24.5 & 25.0 & 24.7 & \\
\hline 12 & 47.3 & 44.0 & 46.0 & \\
\hline$>12$ & 28.2 & 31.0 & 29.3 & \\
\hline Missing, $n$ & 4 & 1 & 5 & \\
\hline $\mathrm{BMI}, \mathrm{kg} / \mathrm{m}^{2}$, mean $(\mathrm{SD})$ & $26.1(5.3)$ & $26.6(4.0)$ & $26.3(4.9)$ & $<.0001$ \\
\hline $\mathrm{BMI}, \mathrm{kg} / \mathrm{m}^{2}$ & & & & $<.0001$ \\
\hline$<25$ & 48.8 & 34.0 & 43.1 & \\
\hline $25-30$ & 30.8 & 48.8 & 37.7 & \\
\hline$>30$ & 20.4 & 17.3 & 19.2 & \\
\hline Missing, $n$ & 11 & 1 & 12 & \\
\hline Smoking status & & & & $<.0001$ \\
\hline Never & 62.5 & 40.3 & 54.0 & \\
\hline Former & 21.4 & 42.7 & 29.6 & \\
\hline Current & 16.1 & 17.0 & 16.4 & \\
\hline Missing, $n$ & & & 0 & \\
\hline Cholesterol no Rx, mg/dL & & & & $<.0001$ \\
\hline$\leq 200$ & 41.3 & 48.2 & 43.9 & \\
\hline $200-239$ & 37.1 & 36.9 & 37.0 & \\
\hline$\geq 240$ & 21.6 & 14.9 & 19.1 & \\
\hline Cholesterol with $\mathrm{Rx}, \mathrm{mg} / \mathrm{dL}$ & & & & $<.0001$ \\
\hline$\leq 200$ & 15.7 & 36.0 & 23.1 & \\
\hline $200-239$ & 43.9 & 36.5 & 41.2 & \\
\hline$\geq 240$ & 40.4 & 27.5 & 35.7 & \\
\hline Blood pressure & & & & $<.0001$ \\
\hline Normal & 30.2 & 15.6 & 24.6 & \\
\hline Prehypertensive & 56.4 & 67.6 & 60.7 & \\
\hline Hypertensive & 13.4 & 16.8 & 14.7 & \\
\hline Missing, $n$ & 5 & 6 & 11 & \\
\hline PPARG rs4684847 & & & & .0910 \\
\hline $\mathrm{CC}$ & 78.8 & 77.3 & 78.2 & \\
\hline $\mathrm{CT} / \mathrm{TT}$ & 21.2 & 22.7 & 21.8 & \\
\hline Missing, $n$ & 188 & 120 & 308 & \\
\hline PPARG rs709158 & & & & .4741 \\
\hline AA & 39.6 & 40.3 & 39.8 & \\
\hline AG/GG & 60.4 & 59.7 & 60.2 & \\
\hline Missing, $n$ & 134 & 98 & 232 & \\
\hline PPARG rs 1175543 & & & & .9218 \\
\hline AA & 40.2 & 40.1 & 40.2 & \\
\hline AG/GG & 59.8 & 59.9 & 59.8 & \\
\hline Missing, $n$ & 136 & 91 & 227 & \\
\hline PPARD rs2016520 & & & & .5240 \\
\hline $\mathrm{CC}$ & 64.6 & 64.0 & 64.4 & \\
\hline $\mathrm{CT} / \mathrm{TT}$ & 35.4 & 36.0 & 35.6 & \\
\hline Missing, $n$ & 146 & 104 & 250 & \\
\hline PPARG rs 1801282 & & & & .2728 \\
\hline Pro/Pro & 78.4 & 77.5 & 78.1 & \\
\hline Pro/Ala or Ala/Ala & 21.6 & 22.5 & 21.9 & \\
\hline Missing, $n$ & 156 & 106 & 262 & \\
\hline
\end{tabular}


TABle 2: The associations between PPAR SNPs and cardiovascular risk factors. Goodness of fit likelihood ratio $P$ value using logistic regression modeling adjusted for age; $P$ value for age based on Student's $t$-test. Combined outcomes had condition at baseline or reported on followup questionnaires (for diabetes, self-reported on followup questionnaires only).

(a)

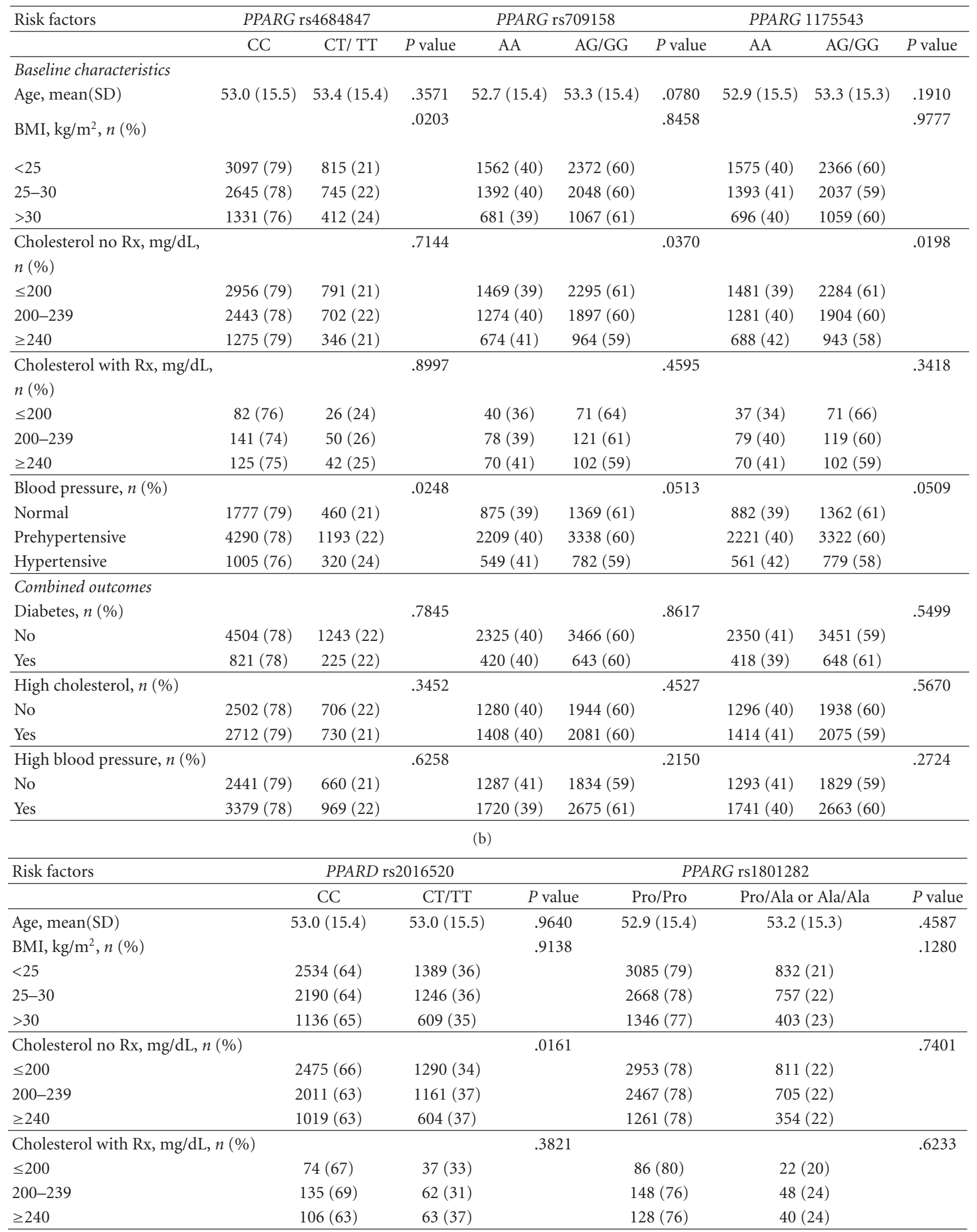


(b) Continued.

\begin{tabular}{|c|c|c|c|c|c|c|}
\hline \multirow[t]{2}{*}{ Risk factors } & \multicolumn{2}{|c|}{ PPARD rs 2016520} & \multicolumn{4}{|c|}{ PPARG rs 1801282} \\
\hline & $\mathrm{CC}$ & $\mathrm{CT} / \mathrm{TT}$ & $P$ value & Pro/Pro & Pro/Ala or Ala/Ala & $P$ value \\
\hline Blood pressure, $n(\%)$ & & & .8076 & & & .2636 \\
\hline Normal & $1441(64)$ & $812(36)$ & & $1769(79)$ & $483(21)$ & \\
\hline Prehypertensive & $3578(65)$ & $1939(35)$ & & $4312(78)$ & $1199(22)$ & \\
\hline Hypertensive & $842(63)$ & $491(37)$ & & $1016(77)$ & $312(23)$ & \\
\hline \multicolumn{7}{|l|}{ Combined outcomes } \\
\hline Diabetes, $n(\%)$ & & & .6185 & & & .1278 \\
\hline No & $3747(65)$ & $2058(35)$ & & $4517(78)$ & $1275(22)$ & \\
\hline Yes & $675(64)$ & $383(36)$ & & $841(80)$ & $212(20)$ & \\
\hline High cholesterol, $n(\%)$ & & & .3178 & & & .3691 \\
\hline No & $2060(64)$ & $1175(36)$ & & $2516(78)$ & $713(22)$ & \\
\hline Yes & $2263(65)$ & $1225(35)$ & & $2732(79)$ & $740(21)$ & \\
\hline High blood pressure, $n(\%)$ & & & .9412 & & & .8331 \\
\hline No & $2006(64)$ & $1121(36)$ & & $2462(78)$ & $676(22)$ & \\
\hline Yes & $2821(64)$ & $1564(36)$ & & $3384(78)$ & $971(22)$ & \\
\hline
\end{tabular}

contrast, females were more likely to have cholesterol levels greater than $200 \mathrm{mg} / \mathrm{dL}$, either with or without cholesterol medication use, than males. There were no gender differences in baseline age or PPAR SNP prevalence.

Table 2 shows the age-adjusted associations between the PPAR SNPs and cardiovascular risk factors examined at baseline and at follow up. After adjustment for age, BMI at baseline was significantly associated with the PPARG rs4684847 SNP such that individuals with at least one of the less common $\mathrm{T}$ alleles were significantly more likely to have a higher $\mathrm{BMI}$ than individuals carrying the CC genotype. Cholesterol level (without medication use) at baseline was significantly associated with the PPARG rs1175543, the PPARG rs709158, and the PPARD rs2016520 SNPs. Specifically, individuals carrying the PPARG rs $1175543 \mathrm{AA}$, the PPARG rs709158 AA, or the PPARD rs2016520 CT or TT genotypes were significantly more likely to be categorized as having baseline cholesterol levels of $240 \mathrm{mg} / \mathrm{dL}$ or greater compared to those with the PPARG rs1175543 AG or GG, the PPARG rs709158 AG or GG, or the PPARD rs2016520 CC genotypes, respectively. Further, participants carrying the PPARG rs4684847 CT or TT genotypes were more likely to be categorized as being prehypertensive or hypertensive at baseline compared to participants carrying the CC genotype. No statistically significantly associations were observed between any of the PPAR sNPs and high blood pressure, high cholesterol, or diabetes diagnoses over the entire followup period. To note, after adjustment for multiple testing, none of the associations between the SNPs and the cardiovascular risk factors were statistically significant.

There were no statistically significant associations between the PPAR SNPs and cardiovascular deaths or events (including nonfatal events combined and MI alone) (see Table 3). A $40 \%$ reduction in the risk of premature cardiovascular death was observed for individuals with the PPARG rs4684847 CT or TT genotype compared to the CC genotype; however, the confidence interval was wide due to a small number of deaths (see Table 4). There were no statis- tically significant associations for premature death (all cause and cardiovascular-related) and PPARG rs709158, PPARG rs1175543, PPARG Pro12Ala, and PPARD rs2016520.

\section{DISCUSSION}

In general, the findings from this prospective, communitybased cohort study indicate that the selected SNPs in PPAR genes are not associated with overall and premature cardiovascular morbidity and mortality. Further, with a few exceptions, we found that the selected PPAR SNPs were not associated with risk factors of cardiovascular disease. These findings were consistent among both men and women, among those with a diagnosis of diabetes, and in strata defined by baseline BMI.

Data on PPAR polymorphisms and cardiovascular disease and cardiovascular disease risk factors are limited and inconsistent. The most studied PPAR polymorphism, PPARG Pro12Ala, has been shown to be associated with reduced PPARG activity [14], and initial studies reported a lower risk of type 2 diabetes associated with the Ala12 allele [14]. This association was observed in some [15-19], but not all [20-23], subsequent studies. The lack of consistency also extends to studies examining the PPARG Pro12Ala polymorphism and cardiovascular disease events: one prospective cohort study examining the PPARG Pro12Ala polymorphism and cardiovascular disease suggested a decreased risk of $\mathrm{CHD}$ among carriers of the Ala12 allele [8], while others have shown no association $[9,10,24]$ or an increase in risk $[9,25]$. Although our study was limited by the small number of cardiovascular events, our results are consistent with those previous studies that have shown no association. This finding, as well as the data showing a lack of association between PPARG Pro12Ala and cardiovascular risk factors, suggests that this SNP is not involved in the development of cardiovascular disease in this population.

While most of the analyses conducted resulted in null findings, statistically significant age-adjusted associations 
TABLE 3: Age-adjusted hazard ratios (HR) and 95\% confidence intervals (95\% CI) of all-cause and cardiovascular mortality and nonfatal cardiovascular events for 5 PPAR polymorphisms. Person years: cumulative person years of follow up. RR (95\% CI): relative risk using poisson regression.

\begin{tabular}{|c|c|c|c|c|c|c|c|c|}
\hline \multirow[b]{2}{*}{ SNPs } & \multirow[b]{2}{*}{ Person years } & \multicolumn{2}{|c|}{ All-cause death } & \multicolumn{2}{|c|}{ CV-related death } & \multicolumn{2}{|c|}{ Nonfatal CV events } & \multirow{2}{*}{$\begin{array}{c}\text { Fatal and nonfatal CV events } \\
\text { RR }(95 \% \mathrm{CI})\end{array}$} \\
\hline & & $n$ & HR (95\% CI) & $n$ & $\operatorname{HR}(95 \% \mathrm{CI})$ & $n$ & RR (95\% CI) & \\
\hline \multicolumn{9}{|l|}{ PPARG rs4684847 } \\
\hline CC & 92827 & 1705 & 1.00 (reference) & 416 & 1.00 (reference) & 2351 & 1.00 (reference) & 1.00 (reference) \\
\hline $\mathrm{CT} / \mathrm{TT}$ & 25880 & 474 & $0.95(0.85-1.05)$ & 114 & $0.90(0.73-1.11)$ & 679 & $1.00(0.73-1.37)$ & $0.88(0.67-1.18)$ \\
\hline \multicolumn{9}{|l|}{ PPARG rs709158 } \\
\hline AA & 47812 & 850 & 1.00 (reference) & 205 & 1.00 (reference) & 1202 & 1.00 (reference) & 1.00 (reference) \\
\hline AG/GG & 71972 & 1328 & $0.99(0.91-1.09)$ & 324 & $1.02(0.86-1.20)$ & 1843 & $0.98(0.75-1.28)$ & $0.98(0.77-1.24)$ \\
\hline \multicolumn{9}{|l|}{ PPARG rs1175543 } \\
\hline AA & 48172 & 867 & 1.00 (reference) & 213 & 1.00 (reference) & 1183 & 1.00 (reference) & 1.00 (reference) \\
\hline AG/GG & 71627 & 1331 & $1.0(0.92-1.09)$ & 316 & $0.99(0.83-1.18)$ & 1864 & $1.03(0.79-1.34)$ & $1.00(0.79-1.28)$ \\
\hline \multicolumn{9}{|l|}{ PPARD rs2016520 } \\
\hline $\mathrm{CC}$ & 77042 & 1386 & 1.00 (reference) & 342 & 1.00 (reference) & 2027 & 1.00 (reference) & 1.00 (reference) \\
\hline $\mathrm{CT} / \mathrm{TT}$ & 42593 & 766 & $1.03(0.94-1.12)$ & 178 & $1.02(0.85-1.23)$ & 1003 & $0.91(0.69-1.19)$ & $0.98(0.76-1.26)$ \\
\hline \multicolumn{9}{|l|}{ PPARG rs1801282 } \\
\hline Pro/Pro & 93254 & 1696 & 1.00 (reference) & 408 & 1.00 (reference) & 2350 & 1.00 (reference) & 1.00 (reference) \\
\hline Pro/Ala or Ala/Ala & 26196 & 456 & $0.96(0.86-1.06)$ & 112 & $0.97(0.79-1.20)$ & 656 & $0.98(0.71-1.35)$ & $0.96(0.72-1.28)$ \\
\hline
\end{tabular}

TABLE 4: Age-adjusted hazard ratios (HR) and 95\% confidence intervals (95\% CI) of premature (age <65 years) all-cause and cardiovascular mortality for 5 PPAR SNPs. Person years: cumulative person years of follow up.

\begin{tabular}{|c|c|c|c|c|c|}
\hline \multirow[b]{2}{*}{ SNPs } & \multirow[b]{2}{*}{ Person years } & \multicolumn{2}{|c|}{ All-cause death } & \multicolumn{2}{|c|}{ CV-related death } \\
\hline & & $n$ & HR $(95 \%$ CI $)$ & $n$ & HR (95\% CI) \\
\hline \multicolumn{6}{|l|}{ PPARG rs4684847 } \\
\hline CC & 43445 & 231 & 1.00 (reference) & 27 & 1.00 (reference) \\
\hline $\mathrm{CT} / \mathrm{TT}$ & 11743 & 58 & $1.05(0.78-1.40)$ & 4 & $0.60(0.21-1.72)$ \\
\hline \multicolumn{6}{|l|}{ PPARG rs709158 } \\
\hline $\mathrm{AA}$ & 23078 & 115 & 1.00 (reference) & 13 & 1.00 (reference) \\
\hline AG/GG & 32822 & 175 & $1.05(0.83-1.33)$ & 18 & $0.95(0.47-1.95)$ \\
\hline \multicolumn{6}{|l|}{ PPARG rs1175543 } \\
\hline $\mathrm{AA}$ & 22875 & 115 & 1.00 (reference) & 14 & 1.00 (reference) \\
\hline AG/GG & 32859 & 175 & $1.04(0.82-1.32)$ & 17 & $0.83(0.41-1.68)$ \\
\hline \multicolumn{6}{|l|}{ PPARD rs 2016520} \\
\hline $\mathrm{CC}$ & 36389 & 186 & 1.00 (reference) & 21 & 1.00 (reference) \\
\hline $\mathrm{CT} / \mathrm{TT}$ & 19940 & 99 & $0.98(0.77-1.25)$ & 9 & $0.79(0.36-1.71)$ \\
\hline \multicolumn{6}{|l|}{ PPARG rs 1801282} \\
\hline Pro/Pro & 44263 & 236 & 1.00 (reference) & 25 & 1.00 (reference) \\
\hline Pro/Ala or Ala/Ala & 12247 & 50 & $0.90(0.66-1.23)$ & 5 & $0.79(0.30-2.09)$ \\
\hline
\end{tabular}

were observed for PPARG rs4684847 with both baseline BMI and blood pressure, and for PPARG rs709158, PPARG rs1175543 and PPARD rs2016520 with baseline cholesterol levels. These associations were not statistically significant when self-reported blood pressure or high cholesterol at any time point over the study period were considered as outcome variables. Further, the statistical significance of the associations disappeared after correction for multiple testing. However, this may be due to a lack of statistical power. To our knowledge, these polymorphisms have not been examined in relation to cardiovascular disease or cardiovascular disease risk factors. Further, the functionalities of these polymorphisms are unknown. Although located in intron regions, these polymorphisms may affect enzyme distribution or other physiological functions related to cardiovascular health. Alternatively, it may be that the associations observed with these SNPs in this study are due to the polymorphisms being in linkage disequilibrium with other functional polymorphisms in the respective regions.

Several limitations of this study must be considered when interpreting the results. First, all of the followup data on cardiovascular risk factors and events were based on self-report. Because of this, there were some missing data for those who did not complete any of the followup questionnaires. 
Further, among those who did complete the questionnaires, there is the possibility of misclassification; this would likely be nondifferential, resulting in a dilution of the true risk estimate. However, in another investigation of this population, self-report of incidence of MI was compared to data gathered from on ongoing county-wide surveillance of MI events documented by hospital review and the sensitivity and specificity of reports were in excess of $94 \%$ (HY Huang, personal communication). A second limitation is that because of the racial homogeneity of the sample we were not able to explore potential racial differences in the associations between the PPAR polymorphisms and cardiovascular disease. In a previous publication [12], we did find, however, racial differences in the prevalence of the PPAR polymorphisms in this study that are consistent with those published in the SNP500 and dbSNP databases.

\section{CONCLUSIONS}

The results from this prospective, community-based cohort study showed no association between the selected PPAR polymorphisms and cardiovascular morbidity and mortality. In addition, in general, no statistically significant associations were observed between the PPAR polymorphisms and cardiovascular risk factors. Future studies should be conducted to confirm these findings and to explore the associations in populations with greater racial and ethnic heterogeneity.

\section{ACKNOWLEDGMENTS}

This study was supported by research Grant 1U01AG18033 from the National Institute on Aging. The authors acknowledge the contributions of Alyce Burke and Judy HoffmanBolton and thank Dr. Sonja Berndt for her review of a draft of the manuscript.

\section{REFERENCES}

[1] W. A. Hsueh and D. Bruemmer, "Peroxisome proliferatoractivated receptor $\gamma$ : implications for cardiovascular disease," Hypertension, vol. 43, no. 2, pp. 297-305, 2004.

[2] R. Bordet, T. Ouk, O. Petrault, et al., "PPAR: a new pharmacological target for neuroprotection in stroke and neurodegenerative diseases," Biochemical Society Transactions, vol. 34, no. 6, pp. 1341-1346, 2006.

[3] J. P. Berger, T. E. Akiyama, and P. T. Meinke, "PPARs: therapeutic targets for metabolic disease," Trends in Pharmacological Sciences, vol. 26, no. 5, pp. 244-251, 2005.

[4] R. M. Touyz and E. L. Schiffrin, "Peroxisome proliferatoractivated receptors in vascular biology-molecular mechanisms and clinical implications," Vascular Pharmacology, vol. 45, no. 1, pp. 19-28, 2006.

[5] N. Marx, H. Duez, J.-C. Fruchart, and B. Staels, "Peroxisome proliferator-activated receptors and atherogenesis: regulators of gene expression in vascular cells," Circulation Research, vol. 94, no. 9, pp. 1168-1178, 2004.

[6] G. Chinetti, S. Griglio, M. Antonucci, et al., "Activation of proliferator-activated receptors $\alpha$ and $\gamma$ induces apoptosis of human monocyte-derived macrophages," Journal of Biological Chemistry, vol. 273, no. 40, pp. 25573-25580, 1998.
[7] S. Cresci, "The PPAR genes, cardiovascular disease and the emergence of PPAR pharmacogenetics," Expert Opinion on Pharmacotherapy, vol. 6, no. 15, pp. 2577-2591, 2005.

[8] P. M. Ridker, N. R. Cook, S. Cheng, et al., "Alanine for proline substitution in the peroxisome proliferator-activated receptor gamma-2 (PPARG2) gene and the risk of incident myocardial infarction," Arteriosclerosis, Thrombosis, and Vascular Biology, vol. 23, no. 5, pp. 859-863, 2003.

[9] T. Pischon, J. K. Pai, J. E. Manson, et al., "Peroxisome proliferator-activated receptor- $\gamma 2$ P12A polymorphism and risk of coronary heart disease in US men and women," Arteriosclerosis, Thrombosis, and Vascular Biology, vol. 25, no. 8, pp. 1654-1658, 2005.

[10] M. Blüher, T. Klemm, T. Gerike, H. Krankenberg, G. Schuler, and R. Paschke, "Lack of association between peroxisome proliferator-activated receptor- $\gamma$ - 2 gene variants and the occurrence of coronary heart disease in patients with diabetes mellitus," European Journal of Endocrinology, vol. 146, no. 4, pp. 545-551, 2002.

[11] J. M. Genkinger, E. A. Platz, S. C. Hoffman, et al., "C47T polymorphism in manganese superoxide dismutase (MnSOD), antioxidant intake and survival," Mechanisms of Ageing and Development, vol. 127, no. 4, pp. 371-377, 2006.

[12] H. Y. Huang, L. Thuita, P. Strickland, S. C. Hoffman, G. W. Comstock, and K. J. Helzlsouer, "Frequencies of single nucleotide polymorphisms in genes regulating inflammatory responses in a community-based population," BMC Genetics, vol. 8, pp. 1-7, 2007.

[13] M. Klintschar and F. Neuhuber, "Evaluation of an alkaline lysis method for the extraction of DNA from whole blood and forensic stains for STR analysis," Journal of Forensic Sciences, vol. 45, no. 3, pp. 669-673, 2000.

[14] S. S. Deeb, L. Fajas, M. Nemoto, et al., "A Pro12Ala substitution in PPAR $\gamma 2$ associated with decreased receptor activity, lower body mass index and improved insulin sensitivity," $\mathrm{Na}$ ture Genetics, vol. 20, no. 3, pp. 284-287, 1998.

[15] D. Altshuler, J. N. Hirschhorn, M. Klannemark, et al., "The common PPAR $y$ Pro12Ala polymorphism is associated with decreased risk of type 2 diabetes," Nature Genetics, vol. 26, no. 1 , pp. 76-80, 2000.

[16] A. S. F. Doney, B. Fischer, J. E. Cecil, et al., "Association of the Pro12Ala and C1431T variants of PPARG and their haplotypes with susceptibility to type 2 diabetes," Diabetologia, vol. 47, no. 3, pp. 555-558, 2004.

[17] H. Mori, H. Ikegami, Y. Kawaguchi, et al., "The Pro ${ }^{12} \rightarrow$ Ala substitution in PPAR- $\gamma$ is associated with resistance to development of diabetes in the general population: possible involvement in impairment of insulin secretion in individuals with type 2 diabetes," Diabetes, vol. 50, no. 4, pp. 891-894, 2001.

[18] A. Memisoglu, F. B. Hu, S. E. Hankinson, et al., "Prospective study of the association between the proline to alanine codon 12 polymorphism in the PPAR $\gamma$ gene and type 2 diabetes," $D i$ abetes Care, vol. 26, no. 10, pp. 2915-2917, 2003.

[19] J. C. Florez, K. A. Jablonski, M. W. Sun, et al., "Effects of the type 2 diabetes-associated PPARG P12A polymorphism on progression to diabetes and response to troglitazone," Journal of Clinical Endocrinology and Metabolism, vol. 92, no. 4, pp. 1502-1509, 2007.

[20] D. Šrámková, M. Kunešová, V. Hainer, M. Hill, J. Vcelák, and B. Bendlová, "Is a Pro12Ala polymorphism of the PPAR $\gamma 2$ gene related to obesity and type 2 diabetes mellitus in the Czech population?" Annals of the New York Academy of Sciences, vol. 967, pp. 265-273, 2002. 
[21] V. I. Lindi, M. I. J. Uusitupa, J. Lindström, et al., "Association of the Pro12Ala polymorphism in the PPAR- $\gamma 2$ gene with 3 -year incidence of type 2 diabetes and body weight change in the Finnish Diabetes Prevention Study," Diabetes, vol. 51, no. 8, pp. 2581-2586, 2002.

[22] R. A. Hegele, H. Cao, S. B. Harris, B. Zinman, A. J. G. Hanley, and C. M. Anderson, "Peroxisome proliferator-activated receptor- $\gamma 2$ P12A and type 2 diabetes in Canadian Oji-Cree," Journal of Clinical Endocrinology and Metabolism, vol. 85, no. 5, pp. 2014-2019, 2000.

[23] M. T. Malecki, J. Frey, T. Klupa, et al., "The Pro12Ala polymorphism of PPAR $\gamma 2$ gene and susceptibility to type 2 diabetes mellitus in a Polish population," Diabetes Research and Clinical Practice, vol. 62, no. 2, pp. 105-111, 2003.

[24] E. J. Rhee, C. H. Kwon, W. Y. Lee, et al., "No association of Pro12Ala polymorphism of PPAR- $\gamma$ gene with coronary artery disease in Korean subjects," Circulation Journal, vol. 71, no. 3, pp. 338-342, 2007.

[25] L. Li, L.-X. Cheng, R. Nsenga, M.-A. He, and T.-C. Wu, "Association between Pro12Ala polymorphism of peroxisome proliferator-activated receptor-gamma 2 and myocardial infarction in the Chinese Han population," Clinical Cardiology, vol. 29, no. 7, pp. 300-304, 2006. 


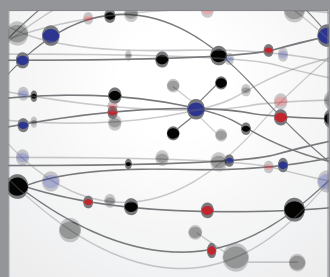

The Scientific World Journal
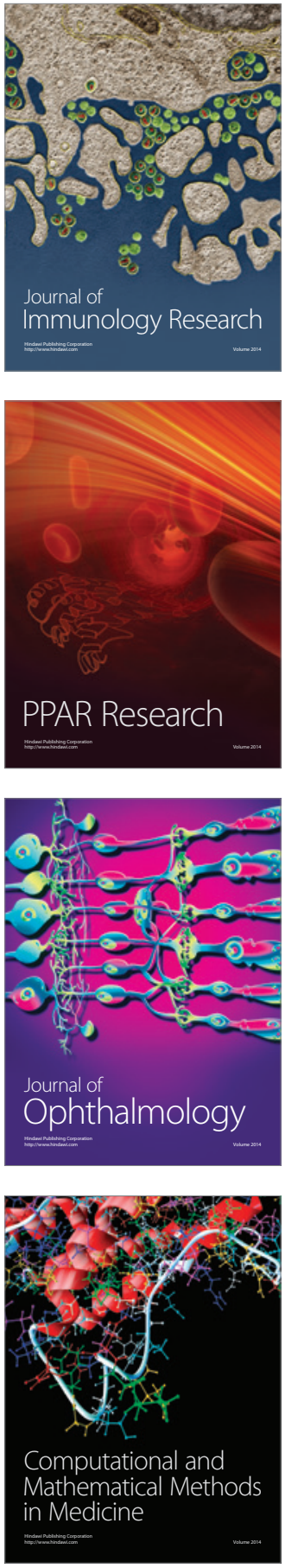

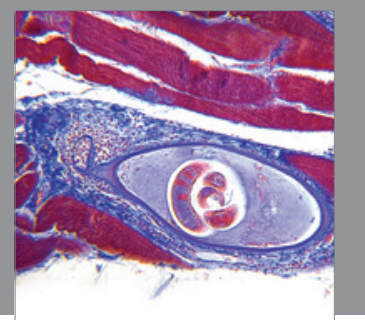

Gastroenterology

Research and Practice
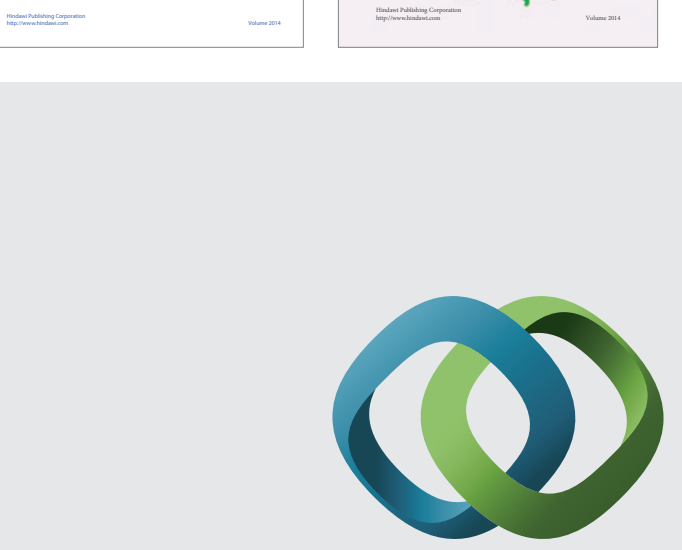

\section{Hindawi}

Submit your manuscripts at

http://www.hindawi.com
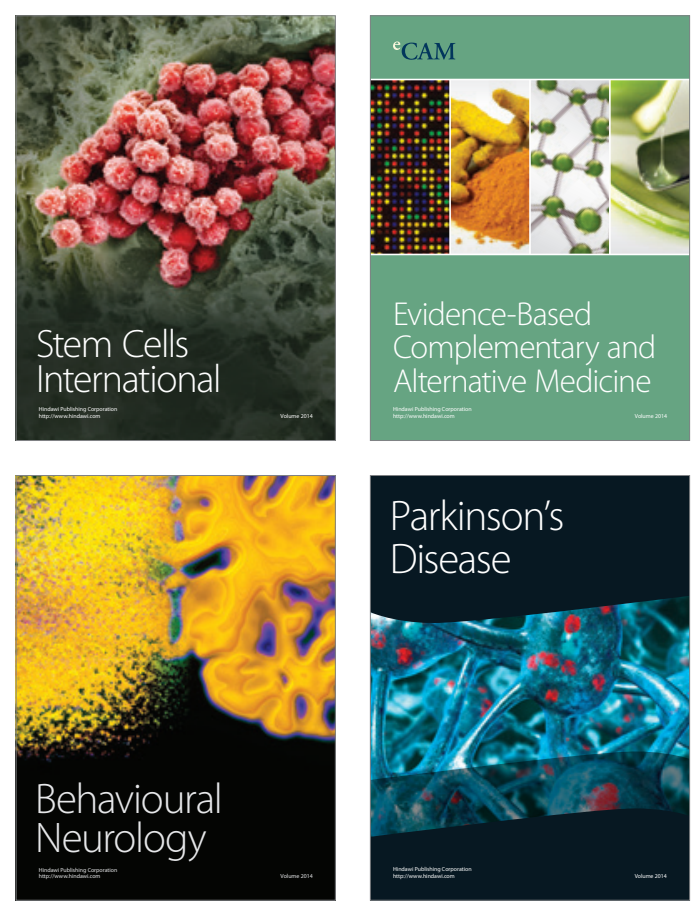

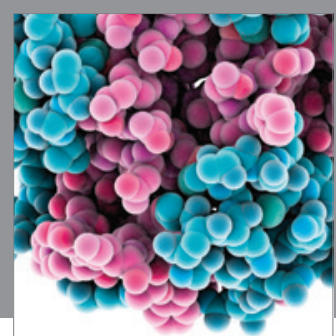

Journal of
Diabetes Research

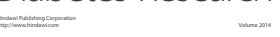

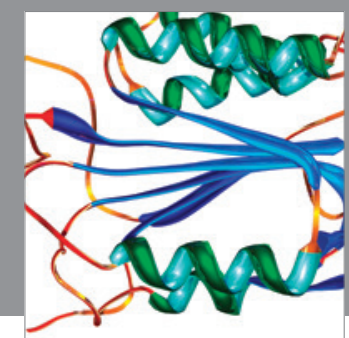

Disease Markers
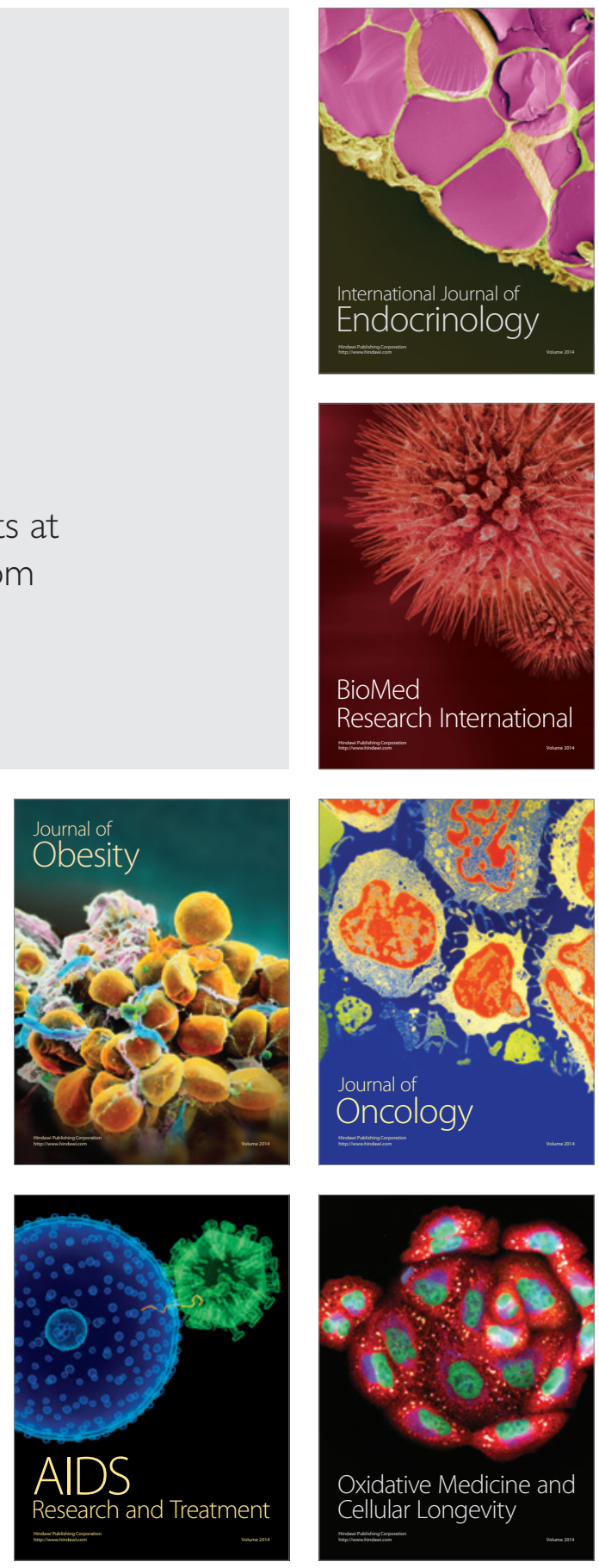